\title{
Editorial
}

\section{Teleradiology: An Internet Cafe Approach to Diagnostics}

\author{
Shraddha Singhania ${ }^{1}$ \\ ${ }^{1}$ Department of Radiodiagnosis, Acharya Vinoba Bhave Rural \\ Hospital, Sawangi, Wardha, Maharashtra, India
}

Int J Recent Surg Med Sci 2019;5:1

Radiodiagnosis is not only the most sought-after speciality in medicine but also an indispensable tool of health care. This has foregrounded certain important issues such as lack of radiologists and radiologic equipment in places requiring the expertise. This state of affairs is worse for subspecialty radiologists who are working mostly in tertiary centers and large academic institutions. These issues are not only limited to India but are faced everywhere in the world. In the United States, there is a persisting shortage of radiologists. In Singapore, there is a scarceness of radiologists for night duties, whereas in the United Kingdom, a radiologist takes 21 days on average to submit a magnetic resonance imaging (MRI) report. ${ }^{1}$

That's where teleradiology comes handy, wherein images are transferred to distant locations for the purpose of interpretation and diagnosis, and with the invention of PACS (picture archiving and communication system) RIS (radiology information system), there is now an efficient and instant communication of images to radiologists and findings to the clinician or surgeon. To meet the increasing demand, teleradiology plays an important role in various ways such as (1) providing diagnostic services to agronomic areas and setups that do not have radiologists at their disposal all the time, (2) hospitals seeking a second opinion, and (3) it also confirms 24-hour availability of radiologists with cost-effectiveness for the administrators.

The practice of teleradiology in India is not very old. A private sector imaging center called Jankharia Imaging in Mumbai successfully used teleradiology in 1996 and the first teleradiology company was Teleradiology Solutions, which was set up in 2002 with its base in Bangalore. ${ }^{1}$ With the current growth of telemedicine, many companies entered this market to provide services for preliminary reads.

However, there are national and international hurdles that are walking hand in hand with the benefits of teleradiology such as (1) despite the growth of medical facilities in India and the reasonably high levels of quality, people in the West still consider us as the third world, which means there are challenges regarding international recognition of medical qualifications in different parts of the world and also many countries have stringent qualification requirement regarding those able to do teleradiology, (2) incompatibility of the teleradiology equipment, (3) resolution problems of imaging visual display units, (4) undecipherable scans due to poor quality of the image, (5) incomplete studies and patchy clinical information, (6) lack of review, etc. There is also a major drawback of teleradiology for radiologists; for example, some companies exploit radiologists by paying peanuts for a highly skilled job and keep the Lion's share for themselves, which in turn results in falling of radiologist's remuneration everywhere.

Many district and rural hospitals have computed tomographic scanners, but radiologists are few and less in number. Most such hospitals are government hospitals and they do not believe in teleradiology solutions. This is despite the request by the former president Late Dr. Abdul Kalam, who advised to take advantage of the benefits of telemedicine in a cost-effective manner in rural areas. ${ }^{2}$ Hence, in conclusion all I would like to mention is that teleradiology as a budding business model should be used to its maximum benefit.

Did you know: Outsourcing of "on-call" night reporting is popularly called "night hawking."

\section{Conflict of Interest}

None declared.

\section{References}

1 Burute N, Jankharia B. Teleradiology: the Indian perspective. Indian J Radiol Imaging 2009;19(1):16-18

2 Kalam Urges for Using Tele-radiology to Help Rural Poor. News on IT in Healthcare. MedIndia; June 30, 2007
Address for correspondence

Shraddha Singhania, MBBS, DMRD,

Department of Radiodiagnosis,

Acharya Vinoba Bhave Rural

Hospital, Sawangi, Wardha 442001,

Maharashtra, India (e-mail:

shraddha2527@gmail.com).
DOI https://doi.org/

$10.1055 / \mathrm{s}-0039-1689062$

ISSN 2455-7420.
(C2019 Medical and Surgical

Update Society
License terms

(1) (1) $\ominus \circledast$ 